Research Article

\title{
Accuracy Improvement Method of a 3D Laser Scanner Based on the D-H Model
}

\author{
Kai Chen (D), ${ }^{1,2}$ Kai Zhan, ${ }^{1,2}$ Xiaocong Yang, ${ }^{1}$ and Da Zhang ${ }^{1}$ \\ ${ }^{1}$ Beijing General Research Institute of Mining and Metallurgy, Building 23, Zone 18 of ABP, No. 188, South 4th Ring Road West, \\ Beijing 102628, China \\ ${ }^{2}$ School of Mechanical Engineering, University of Science and Technology Beijing, No. 30 Xueyuan Road, Haidian District, \\ Beijing 100083, China \\ Correspondence should be addressed to Kai Chen; chenkai@bgrimm.com
}

Received 11 March 2021; Revised 9 April 2021; Accepted 5 May 2021; Published 25 May 2021

Academic Editor: Yuantian Sun

Copyright (C) 2021 Kai Chen et al. This is an open access article distributed under the Creative Commons Attribution License, which permits unrestricted use, distribution, and reproduction in any medium, provided the original work is properly cited.

\begin{abstract}
A three-dimensional (3D) laser scanner with characteristics such as acquiring huge point cloud data and noncontact measurement has revolutionized the surveying and mapping industry. Nonetheless, how to guarantee the 3D laser scanner precision remains the critical factor that determines the excellence of 3D laser scanners. Hence, this study proposes a 3D laser scanner error analysis and calibration-method-based D-H model, applies the D-H model method in the robot area to the 3D laser scanner coordinate for calculating the point cloud data and creatively derive the error model, comprehensively analyzes six external parameters and seven inner structure parameters that affect point cloud coordinator error, and designs two calibration platforms for inner structure parameters. To validate the proposed method, we used SOKKIA total station and BLSS-PE 3D laser scanner to attain the center coordinate of the testing target sphere and then evaluate the external parameters and modify the point coordinate. Based on modifying the point coordinate, comparing the point coordinate that considered the inner structure parameters with the point coordinate that did not consider the inner structure parameters, the experiment revealed that the BLSS-PE 3D laser scanner's precision enhanced after considering the inner structure parameters, demonstrating that the error analysis and calibration method was correct and feasible.
\end{abstract}

\section{Introduction}

Three-dimensional (3D) laser scanning technology [1] is a new spatial data acquisition technology that has revolutionized the surveying and mapping industry. Compared with the $3 \mathrm{D}$ photogrammetry and triangle photogrammetry technology, 3D laser scanning technology offers advantages like high measurement accuracy, simple data processing, and broad range. Meanwhile, 3D laser scanning technology is a cost-effective and practical solution for $3 \mathrm{D}$ measurements $[2,3]$ because of a low-energy laser pulse, strong ability to resist outside light interference, and no effect on the human eye.

While using a 3D laser scanner, the precision and index of the instrument have strict requirements, and how to ensure the precision of the $3 \mathrm{D}$ laser scanner that can fulfill the actual use of requirements is imperative. Currently, many scientific research institutions are researching the theory and technology of 3D laser scanning and have made some achievements. A study [4] explored the factors affecting the performance of a 3D laser scanner from the aspects of measuring distance, the color of the object to be measured, and laser incident angle. Another study [5] assessed the quality of the data collected by the ground $3 \mathrm{D}$ laser scanner through experiments and obtained the plane coordinates and elevation accuracy of the scanning point of the system. In addition, a study [6] performed theoretical analysis on multiple factors affecting the measurement error of a 3D laser scanner. Furthermore, the measurement accuracy of the Riegl LMS-Q140I-80 3D laser scanner was evaluated in another study [7], and the experimental results revealed that the actual measurement accuracy corroborated 
the nominal measurement accuracy. All the studies mentioned above primarily investigated the measurement performance and precision of a 3D laser scanner.

In recent years, some scholars started using the selfchecking calibration method to validate the system error of 3D laser scanners. A study [8] assumed that the system error type of the ground $3 \mathrm{D}$ laser scanner was similar to that of the total station and, thus, proposed the concept of the 3D laser scanner calibration model, including nine parameters such as rotation angle, translation between three shafts of a $3 \mathrm{D}$ laser scanner, and instrument addition and multiplication constant. In addition, a study [9] proposed an additional seven-parameter model and the self-checking process of the $3 \mathrm{D}$ free network by using the theodolite error model, as well as executed the checking of the Faro 880 3D laser scanner. Another study [10] enhanced the error model previously constructed and reconstructed the mathematical model with 19 additional parameters. A study [11] explored the impact of several crucial factors and their control methods on the accuracy of Leica's HDS 3D laser scanner. A study [12] analyzed the influence of six error factors on the coordinates of scanning points through a theoretical simulation test and then validated the detection model with simulated numerical values. Moreover, a study [13] built a scanner self-checking model with 10 parameters, examined three 3D laser scanners, and attained the ranging error, horizontal angle error, and vertical angle error of 3D laser scanners. A study [14] analyzed the influence of the positioning error of an airborne LIDAR system on the positioning accuracy and determined the errors of the positioning parameters among the GPS, laser scanner, and inertial navigation unit by using the selfchecking calibration method. Furthermore, a study [15] summarized the determination method of the $3 \mathrm{D}$ laser scanning system error and proposed a self-check calibration method to calibrate the instrument system error. To date, no uniform standard exists for the parameters in the selfchecking method.

Starting from the working principle and instrument structure of a $360^{\circ}$ laser scanner developed by SureStar Technology Co., Ltd., a study [16] comprehensively analyzed the source of angle measurement error of such equipment, emphatically analyzed the impact of installation eccentricity of the photoelectric encoder on the system's measurement accuracy, and deduced and established the angle error model. Based on the correlation between position and angle of each sensor in the vehicle-mounted 3D laser scanner system developed by Wuhan University, a study [17] proposed the concept of combined check and calibration of the vehicle-mounted mobile measurement system. The combined check and calibration of the laser ranging sensor and IMU are primarily implemented by using the check and calibration method based on the control point and feature surface. To date, some studies have considered the impact of the internal structure of the 3D laser scanner or sensor installation on measurement error; however, there is a lack of an overall and essential error analysis model.

The error analysis of the $3 \mathrm{D}$ laser scanner primarily analyzed the impact of the external influence factors on the measurement error, using the method of mathematical modeling, self-calibration, or combination calibration method for system calibration-all these methods [18, 19] can enhance the system accuracy to a certain extent; however, these methods just partially considered the influence on the equipment's accuracy due to the system's internal structure. This study innovatively introduces the D-H modeling method of robotics into the measurement error analysis [20, 21] and derives the error influencing factors of the $3 \mathrm{D}$ laser scanner, including the influence of external parameters and internal structure parameters on the measurement accuracy. To ensure the measurement accuracy of the system to the maximum extent, two sets of internal parameter calibration platforms are designed. To validate the system's accuracy improvement effect, the high-precision measurement method is used for verification, and the test results demonstrate the method's efficacy.

\section{D-H Error Model Establishment and Analysis}

2.1. 3D Laser Scanning Principle. The fundamental principle of the 3D laser scanner is that, first, a drive scanner is used to rotate in the axial direction by the axial motor and the axial rotation angle $\alpha$ is obtained by using an angle encoder and, second, a drive scanner is used to rotate in the radial direction by the radial motor and the radial rotation angle $\beta$ is obtained by using an angle encoder; at the same time, the laser sensor measures the fly time and evaluates the distance $S$ to the target. The point $P$ space coordinates (shown in Figure 1) calculating formula is as follows:

$$
\left\{\begin{array}{l}
X_{p}=S \cdot \cos \beta \cdot \cos \alpha, \\
Y_{p}=S \cdot \cos \beta \cdot \sin \alpha, \\
Z_{p}=S \cdot \sin \beta .
\end{array}\right.
$$

Distance $S$ calculating formula is $S=(1 / 2) c \cdot t$, where $c$ is the speed at which a laser travels through the atmosphere and $t$ is the round-trip time of laser flying.

\subsection{Error Formula Derivation}

2.2.1. D-H Model Establishment Method. D-H modeling method is a standard method to represent robots and model robot motions. Assumedly, the robot comprises a series of joints and linkages, which could be sliding or rotating and can be placed in any order and any plane. First, a reference coordinate system is assigned to each joint. Then, the steps for the transformation from one joint to the next are determined. Second, all transformations from the base to the first joint and then from the first joint to the second joint until to the last joint are combined to obtain the total transformation matrix of the robot. Figure 2 shows the D-H modeling method.

\subsubsection{Calculating Model Establishment of Point Cloud.} The 3D laser scanning principle presents the formula of how to calculate the coordinates of spatial point clouds. The formula is derived on the basis of the simplified model, which only considers the measurement distance $S$, axial 


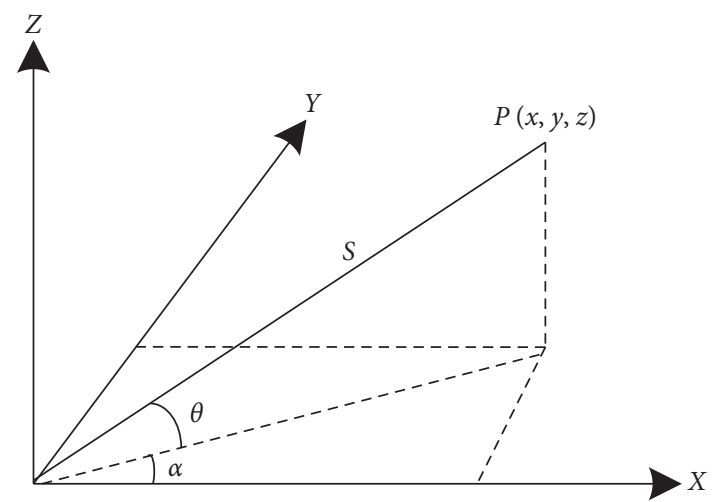

FIgURE 1: The point coordinates in the coordinate system of the 3D laser scanner.

scanning angle $\alpha$, and radial scanning angle $\beta$, but many internal structural factors are not considered. The D-H modeling method is introduced to entirely derive the coordinate solution formula of the point cloud space.

The following definitions are given to fulfill the D-H model establishment principle:

Point $P$ is assumed as the target point of the measured object

Point $Z$ is assumed to be zero of the laser ranging sensor Point $\mathrm{O}_{0}$ is assumed as the intersection point of the end face of the cross target and target laser axis

Point $O_{1}$ is assumed as the intersection point of the end face of the cross target and axial motor axis

Point $\mathrm{O}_{2}$ is assumed as the intersection point of the axial motor axis and radial motor axis

Point $\mathrm{O}_{3}$ is assumed as the intersection point of the radial motor axis and measurement face of the laser sensor

$\mathrm{O}_{0}, \mathrm{O}_{1}, \mathrm{O}_{2}$, and $\mathrm{O}_{3}$ are assumed as the origin, and coordinate system 0 , coordinate system 1 , coordinate system 2, and coordinate system 3 are established successively

The measuring distance of the laser ranging module is assumed as $d$, axial rotation angle as $\alpha$, radial rotation angle as $\beta$, the distance from the laser ranging distance sensor zero point to the $Y Z$ plane of the coordinate system as L1, the distance from the laser ranging distance sensor zero point to the $X Z$ plane of the coordinate system as L2, the distance from the coordinate system 1 origin $O_{1}$ to the coordinate system 3 origin $O_{3}$ as $L_{3}$, the distance from the coordinate system 0 origin $\mathrm{O}_{0}$ to the coordinate system 2 origin $\mathrm{O}_{2}$ as $L_{4}$, and the distance from the coordinate system 0 origin $O_{0}$ to the coordinate system 1 origin $O_{1}$ as $L_{5}$

$a_{i-1}$ is assumed as the measurement distance from $Z_{i-1}$ to $Z_{i}$ in the direction $X_{i-1}, \alpha_{i-1}$ as the rotation angle from $Z_{i-1}$ to $Z_{i}$ in the direction, $X_{i-1}, d_{i}$ as the measurement distance from $X_{i-1}$ to $X_{i}$ in the direction $Z_{i}$, and $\vartheta_{i}$ as the rotation angle from $X_{i-1}$ to $X_{i}$ in the direction $Z_{i}$

Figure 3 shows the internal structure of the 3D laser scanner and the relevant definitions of the $\mathrm{D}-\mathrm{H}$ modeling auxiliary coordinate system.

Table 1 lists the D-H modeling parameters.

The homogeneous coordinate transformation matrix between coordinate system $\{i\}$ and coordinate system $\{i-1\}$ is

$$
{ }_{i-1}^{i} T=\left|\begin{array}{cccc}
\cos \vartheta_{i} & -\sin \vartheta_{i} & 0 & a_{i-1} \\
\sin \vartheta_{i} \cos \alpha_{i-1} & \cos \vartheta_{i} \cos \alpha_{i-1} & -\sin \alpha_{i-1} & -\sin \alpha_{i-1} d_{i} \\
\sin \vartheta_{i} \sin \alpha_{i-1} & \cos \vartheta_{i} \sin \alpha_{i-1} & \cos \alpha_{i-1} & \cos \alpha_{i-1} d_{i} \\
0 & 0 & 0 & 1
\end{array}\right| .
$$

We assume any point $P^{3}=\left[\begin{array}{llll}X 3 & Y 3 & Z 3 & 1\end{array}\right] * T$ in the coordinate system 3 , and then, the $3 \mathrm{D}$ coordinate calculating formula of any point $P^{0}$ of coordinate system 0 is as follows:

$$
P^{0}=\prod_{i=1}^{3}\left|\begin{array}{cccc}
\cos \vartheta_{i} & -\sin \vartheta_{i} & 0 & a_{i-1} \\
\sin \vartheta_{i} \cos \alpha_{i-1} & \cos \vartheta_{i} \cos \alpha_{i-1} & -\sin \alpha_{i-1} & -\sin \alpha_{i-1} d_{i} \\
\sin \vartheta_{i} \sin \alpha_{i-1} & \cos \vartheta_{i} \sin \alpha_{i-1} & \cos \alpha_{i-1} & \cos \alpha_{i-1} d_{i} \\
0 & 0 & 0 & 1
\end{array}\right| \begin{gathered}
x_{3} \\
y_{3} \\
z_{3} \\
1
\end{gathered} \mid .
$$

$L_{1}, L_{2}, L_{3}, L_{4}$, and $L_{5}$ are the fixed distance, known quantity; $d$ is the measurement distance of the laser sensor, $\alpha$ is the rotation angle of the radial motor, and $\beta$ is the rotation angle of the axial motor. Table 2 shows the $\mathrm{D}-\mathrm{H}$ modeling parameter.

The coordinate of point $P$ is $P^{3}=\left(\begin{array}{llll}d+L_{1} & L_{2} & 0 & 1\end{array}\right) * T$ in coordinate system 3 , which is the laser sensor coordinate system.

The coordinate of point $P$ in coordinate system 0 , which is the $3 \mathrm{D}$ laser scanner coordinate system, is shown as follows:

$$
P^{0}=\prod_{i=1}^{3}{ }_{i-1}^{i} T P^{3}=\left|\begin{array}{c}
-\left(d+L_{1}\right) \cos \alpha+L_{2} \sin \alpha+L_{4} \\
-\left(d+L_{1}\right) \sin \alpha \cos \beta-L_{2} \cos \alpha \cos \beta-L_{3} \sin \beta \\
\left(d+L_{1}\right) \sin \alpha \sin \beta+L_{2} \cos \alpha \sin \beta-L_{3} \cos \beta-L_{5} \\
1
\end{array}\right|
$$

2.2.3. Point Cloud Error Formula Derivation. The 3D laser scanner point cloud calculation formula contains two types of parameters. One is the transformation parameter of the scanner coordinate system and the external coordinate 


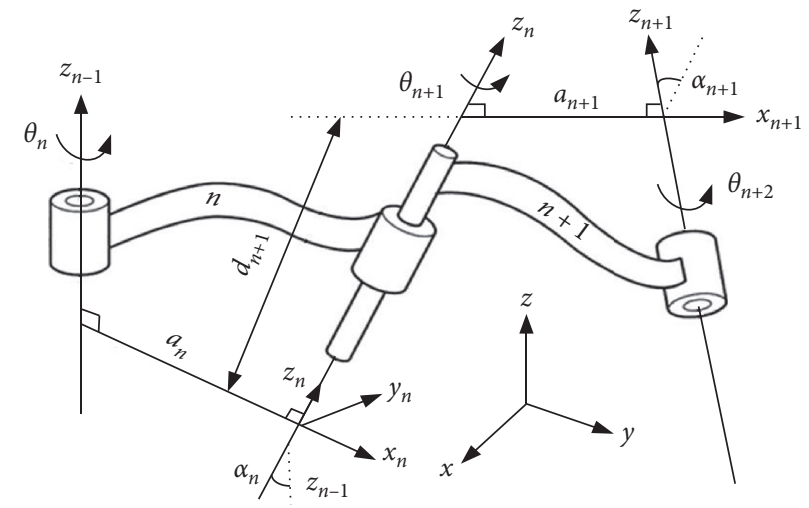

Figure 2: The schematic diagram of the D-H modeling method.

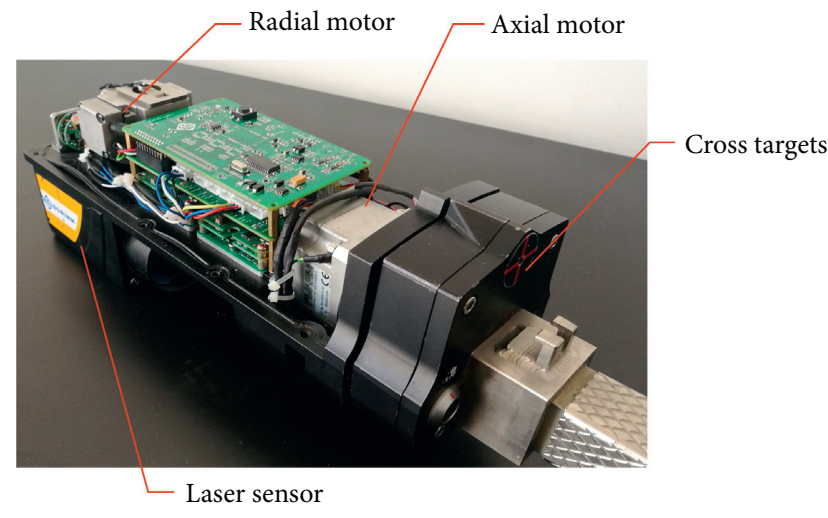

(a)

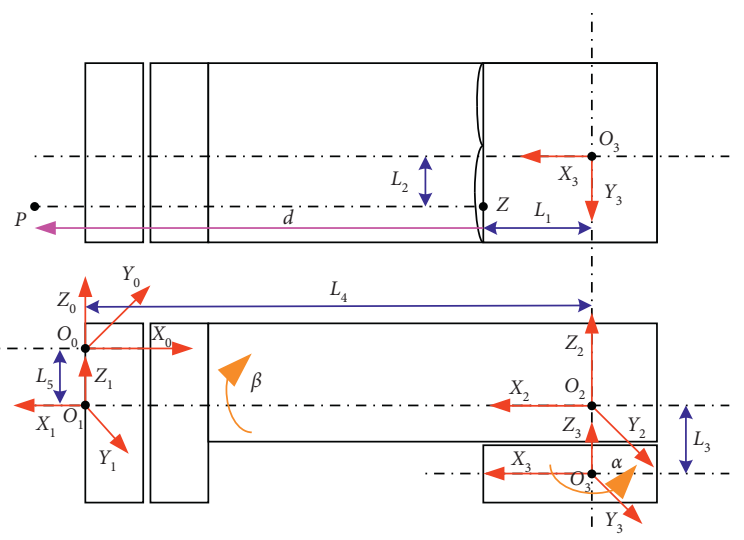

(b)

FIgURE 3: The inner structure of the 3D laser scanner and coordinate system for the D-H model.

system, which is called the external orientation parameter and comprises three translation parameters $(\Delta X, \Delta Y, \Delta Z)$ and three rotation parameters $(I, K, H)$. The other is the internal error parameters of the instrument; the corresponding distance measurement error of parameter $L_{1}$ is $L_{1}^{\rho}$, the corresponding distance measurement error of parameter $L_{2}$ is $L_{2}^{\rho}$, the corresponding distance measurement error of parameter $L_{3}$ is $L_{3}^{\rho}$, the corresponding distance measurement error of parameter $L_{4}$ is $L_{4}^{\rho}$, the corresponding distance measurement error of parameter $L_{5}$ is $L_{5}^{\rho}$, the corresponding rotation angle error of parameter $\alpha$ is $\alpha^{\rho}$, and the corresponding rotation angle error of parameter $\beta$ is $\beta^{\rho}$. In addition, set $(X, Y, Z)$ and $(x, y, z)$ are the coordinates of any point in the external coordinate system and the coordinates in the scanner coordinate system, respectively. Then, the following formula is satisfied between the external coordinate points and the coordinate points in the scanner coordinate system:

$$
F=\left|\begin{array}{c}
X \\
Y \\
Z \\
1
\end{array}\right|=R(I, K, H, 1)\left|\begin{array}{c}
x \\
y \\
z \\
1
\end{array}\right|+\left|\begin{array}{c}
\Delta X \\
\Delta Y \\
\Delta Z \\
1
\end{array}\right|
$$

According to formula (4) and system error of the 3D laser scanner, the coordinate calculating formula of point $P$ in the scanner coordinate system is shown follows:

$$
P^{0}=\left|\begin{array}{l}
x \\
y \\
z \\
1
\end{array}\right|=\left|\begin{array}{c}
-\left(d+L_{1}+L_{1}^{\rho}\right) \cos \Delta \alpha+\alpha^{\rho} \Delta+\left(L_{2}+L_{2}^{\rho}\right) \sin \Delta \alpha+\alpha^{\rho} \Delta+\left(L_{4}+L_{4}^{\rho}\right) \\
-\left(d+L_{1}+L_{1}^{\rho}\right) \sin \Delta \alpha+\alpha^{\rho} \Delta \cos \left(\beta+\beta^{\rho}\right)-\Delta L_{2}+L_{2}^{\rho} \Delta \cos \Delta \alpha+\alpha^{\rho} \Delta \cos \left(\beta+\beta^{\rho}\right)-\Delta L_{3}+L_{3}^{\rho} \Delta \sin \left(\beta+\beta^{\rho}\right) \\
\sin \Delta \alpha+\alpha^{\rho} \Delta \sin \left(\beta+\beta^{\rho}\right)+\Delta L_{2}+L_{2}^{\rho} \Delta \cos \Delta \alpha+\alpha^{\rho} \Delta \sin \left(\beta+\beta^{\rho}\right)-\Delta L_{3}+L_{3}^{\rho} \Delta \cos \left(\beta+\beta^{\rho}\right)-\Delta L_{5}+L_{5}^{\rho} \Delta \\
1
\end{array}\right| .
$$




\section{Initial Value Determination for an Unknown Parameter}

3.1. Initial Value Determination for an External Parameter. The external orientation parameter contains three translation parameters $(\Delta X, \Delta Y, \Delta Z)$ and three rotation parameters $(I, K, H)$. To evaluate the initial value of the external orientation parameter, we pick two pairs of homonymy points $\Delta X_{1}, Y_{1}, Z_{1} \Delta, \Delta x_{1}, y_{1}, z_{1} \Delta$ and $\Delta X_{2}, Y_{2}, Z_{2} \Delta$, $\Delta x_{2}, y_{2}, z_{2} \Delta$, which should satisfy formula (5):

$$
\left.\begin{array}{l}
\left|\begin{array}{c}
X_{1} \\
Y_{1} \\
Z_{1} \\
1
\end{array}\right|=R(I, K, H, 1)\left|\begin{array}{c}
x_{1} \\
y_{1} \\
z_{1} \\
1
\end{array}\right|+\left|\begin{array}{c}
\Delta X \\
\Delta Y \\
\Delta Z \\
1
\end{array}\right| \\
\left|\begin{array}{c}
X_{2} \\
Y_{2} \\
Z_{2} \\
1
\end{array}\right|=R(I, K, H, 1)\left|\begin{array}{c}
x_{2} \\
y_{2} \\
z_{2} \\
1
\end{array}\right|+\left|\begin{array}{c}
\Delta X \\
\Delta Y \\
\Delta Z \\
1
\end{array}\right|
\end{array}\right\} .
$$

The result using the abovementioned equation minus the equation below is

$$
\left|\begin{array}{c}
X_{1}-X_{2} \\
Y_{1}-Y_{2} \\
Z_{1}-Z_{2} \\
1
\end{array}\right|=R(I, K, H, 1)\left|\begin{array}{c}
x_{1}-x_{2} \\
y_{1}-y_{2} \\
z_{1}-z_{2} \\
1
\end{array}\right|
$$

Based on the characteristics of the orthogonal matrix and formula (8), calculating the three angle elements $(I, K, H)$, the translation $(\Delta X, \Delta Y, \Delta Z)$ can be evaluated by substituting them into any formula in equation (7), and the parameter $(\Delta X, \Delta Y, \Delta Z)$ can be taken as the initial value of the external orientation parameter.

\subsection{Initial Value Determination for an Internal Parameter}

3.2.1. Calculating the Internal Distance Parameter. Based on formula (6), there are primarily seven internal parameters of the 3D laser scanner, $L_{1}^{\rho}, L_{2}^{\rho}, L_{3}^{\rho}, L_{4}^{\rho}, L_{5}^{\rho}, \alpha^{\rho}$, and $\beta^{\rho}$, and the seven internal parameters include distance error and angle error. The distance parameter value was obtained according to the design drawing in the theory case; however, the actual situation is the scanner processing structure and assembly process inevitably introduced errors, so using the value of the design drawing was not desirable. In this study, an internal distance parameter calibration platform was designed, including a high-precision clamping tool and a digital level (Figure 4). The Vernier caliper was used to precisely measure the real value $L_{1}^{\wedge}, L_{2}^{\wedge}, L_{3}^{\wedge}, L_{4}^{\wedge}$, and $L_{5}^{\wedge}$ of parameters $L_{1}, L_{2}, L_{3}, L_{4}$, and $L_{5}$. Then, using the equation $L^{\rho}=L-L^{\wedge}$ to calculate the distance error, the result was $L_{1}^{\rho}=0.002 \mathrm{~m}, L_{2}^{\rho}=0.003 \mathrm{~m}, L_{3}^{\rho}=-0.002 \mathrm{~m}, L_{4}^{\rho}=-0.004 \mathrm{~m}$, and $L_{5}^{\rho}=0.004 \mathrm{~m}$.
TABLE 1: D-H model parameters.

\begin{tabular}{lcccc}
\hline Number & $a_{i-1}$ & $\alpha_{i-1}$ & $d_{i-1}$ & $\vartheta_{i}$ \\
\hline 1 & $a_{0}$ & $\alpha_{0}$ & $d_{1}$ & $\vartheta_{1}$ \\
2 & $a_{1}$ & $\alpha_{1}$ & $d_{2}$ & $\vartheta_{2}$ \\
3 & $a_{2}$ & $\alpha_{2}$ & $d_{3}$ & $\vartheta_{3}$ \\
\hline
\end{tabular}

TABle 2: The D-H model real parameters.

\begin{tabular}{lcccc}
\hline Number & $a_{i-1}$ & $\alpha_{i-1}$ & $d_{i-1}$ & $\vartheta_{i}$ \\
\hline 1 & 0 & 0 & $-L_{5}$ & $-180^{\circ}$ \\
2 & $-L_{4}$ & $\beta$ & 0 & 0 \\
3 & 0 & 0 & $-L_{3}$ & $\alpha$ \\
\hline
\end{tabular}

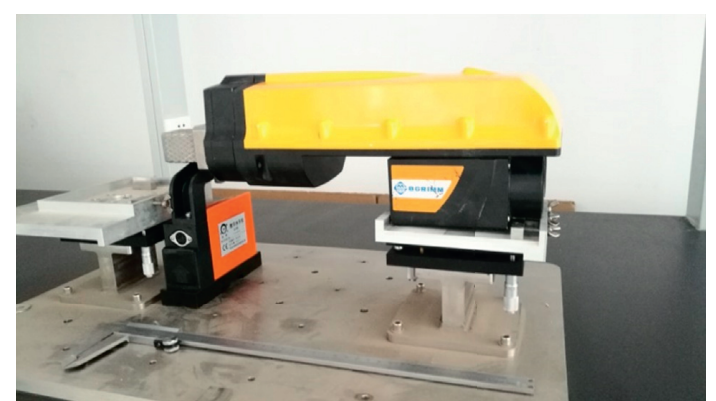

FIgURE 4: The distance parameter calibration platform of the inner structure.

3.2.2. Calculating the Internal Angle Parameter. In this study, we designed the internal angle parameter calibration platform, including high-precision linear guide, high-precision clamping tool, and calibration coordinate paper (as shown in Figure 5). By moving the 3D laser scanner on the linear guide rail, the $2 n$ sets coordinate values $\left(x_{1 i}, y_{1 i}\right) i \in(1,2, \ldots, n)$ and $\left(x_{2 i}, y_{2 i}\right) i \in(1,2, \ldots, n)$ of laser indicators corresponding to the axial direction and radial direction on the calibration coordinate paper were collected, and two lines $y=a_{1}+b_{1} x$ and $y=a_{2}+b_{2} x$ were fitted by the least-square formula:

$$
\left\{a=\bar{y}-b \bar{x}, \quad n x=\sum_{i=1}^{n} x_{i}, n \bar{y}=\sum_{i=1}^{n} y_{i}, b=\frac{\sum_{i=1}^{n} x_{i} y_{i}-\bar{y} \sum_{i=1}^{n} x_{i}}{\sum_{i=1}^{n} x_{i}^{2}-\bar{x} \sum_{i=1}^{n} x_{i}} .\right.
$$

The internal angle parameters $\alpha^{\rho}=2.10^{\circ}$ and $\beta^{\rho}=4.60^{\circ}$ were precisely obtained by calculating the intersection angle between fitting the line $y=a_{1}+b_{1} x, y=a_{2}+$ $b_{2} x$ (as shown in Table 3 ), and the vertical line of the calibrated coordinate paper.

\section{The Result of Error Verification}

4.1. Calculating the External Orientation Parameter. We used a mine 3D laser scanner named BLSS-PE to calculate external orientation parameters; six standard test target balls were set up in the laboratory, the entire laboratory scene was scanned with the $3 \mathrm{D}$ laser scanner to extract the cloud data of test target ballpoints, and the central coordinates of the 


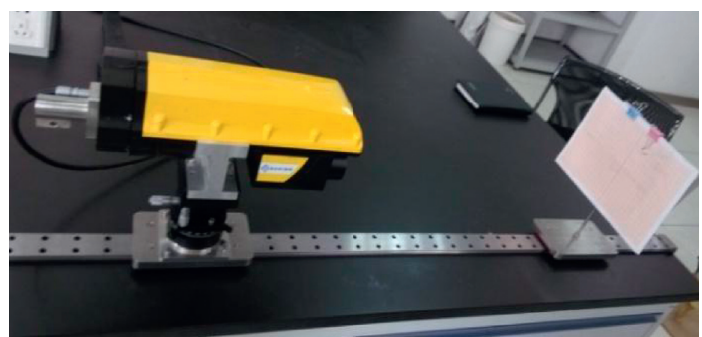

FIGURE 5: The angle parameter calibration platform of the inner structure.

TABLE 3: Calculating the fitting line parameter using the least-square method.

\begin{tabular}{lccccccc}
\hline Number & $x_{1 i}$ & $y_{1 i}$ & $x_{2 i}$ & $y_{2 i}$ & $a_{1}$ & $b_{1}$ & $a_{2}$ \\
\hline 1 & 0.058 & 0.081 & 0.071 & 0.072 & & & \\
2 & 0.065 & 0.079 & 0.073 & 0.074 & & & \\
3 & 0.067 & 0.082 & 0.074 & 0.072 & 0.078 & 0.035 & 0.068 \\
4 & 0.069 & 0.078 & 0.077 & 0.073 & & & \\
5 & 0.073 & 0.082 & 0.080 & 0.075 & & & \\
\hline
\end{tabular}

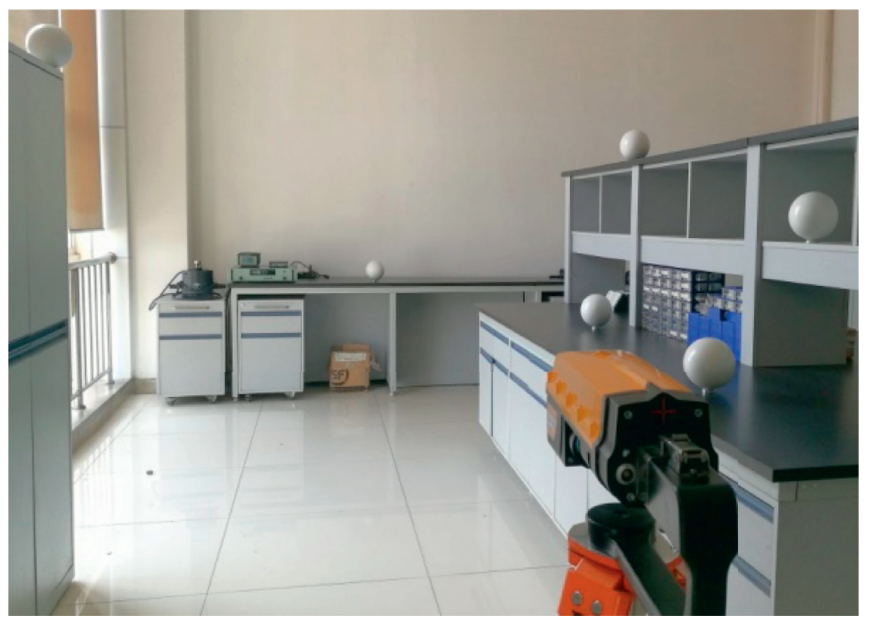

(a)

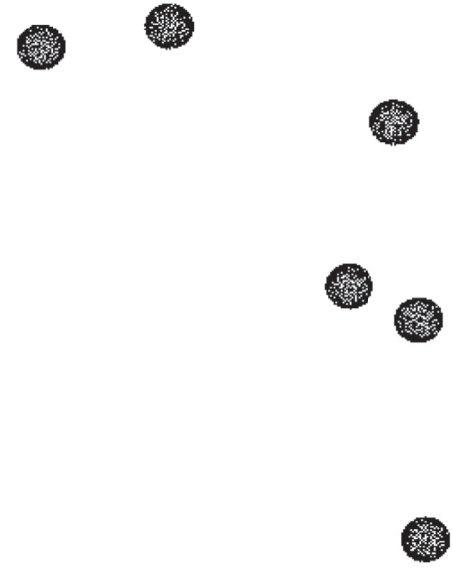

(b)

FIgURE 6: Scanning the test target sphere using the 3D laser scanner.

TABLE 4: The real coordinate of the test target.

\begin{tabular}{lcccccc}
\hline \multirow{2}{*}{ Target ball } & \multicolumn{3}{c}{$\begin{array}{c}\text { Measured value of the } \\
\text { total station }(\mathrm{m})\end{array}$} & \multicolumn{3}{c}{ 3easured value of the } \\
& $X$ & $Y$ & $Z$ & $X$ & $Y$ & $Z$ \\
\hline 1 & 7.181 & 5.083 & 14.408 & 5.008 & 6.054 & 3.052 \\
2 & 7.198 & 4.738 & 14.406 & 4.675 & 6.047 & 3.738 \\
3 & 8.025 & 3.850 & 13.936 & 3.664 & 6.037 & 3.858 \\
4 & 7.294 & 3.896 & 13.560 & 3.556 & 5.884 & 3.057 \\
5 & 7.483 & 2.679 & 12.375 & 1.869 & 5.465 & 3.039 \\
6 & 8.852 & 4.598 & 13.232 & 3.995 & 4.931 & 4.395 \\
\hline
\end{tabular}

target ball were fitted with the software of the 3D laser scanner (as shown in Figure 6). Meanwhile, the total station was used to measure the coordinates of six standard test target balls, and the center coordinates of the target balls fitted by the $3 \mathrm{D}$ laser scanner and the coordinates measured by the total station were used as homonyms to calculate the external orientation parameters (as shown in Figure 7).

Table 4 shows the measurement coordinates of the total station and the 3D laser scanner.

Using the coordinate values of the first four targets and then using formulas (7) and (8), the external orientation parameters are calculated as follows: 


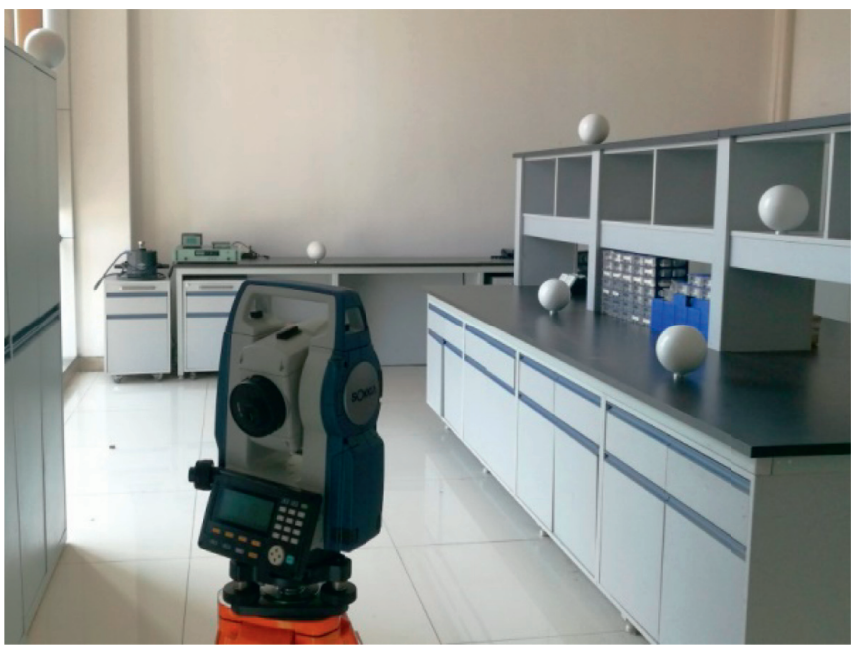

(a)

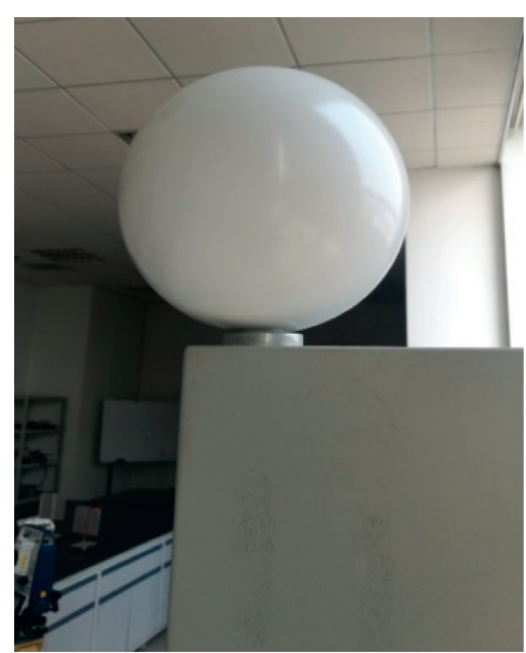

(b)

Figure 7: Scanning the test target sphere using the total station.

TABLE 5: The difference between real and transformed coordinates.

\begin{tabular}{|c|c|c|c|c|c|c|c|c|c|}
\hline \multirow[t]{2}{*}{ Target ball } & \multicolumn{3}{|c|}{$\begin{array}{l}\text { Measured value of the total } \\
\text { station }(\mathrm{m})\end{array}$} & \multicolumn{3}{|c|}{ Correction coordinate $(\mathrm{m})$} & \multicolumn{3}{|c|}{ Difference of coordinate $(\mathrm{m})$} \\
\hline & $X$ & Y & $Z$ & $X$ & $Y$ & $Z$ & $\delta X$ & $\delta Y$ & $\delta Z$ \\
\hline 5 & 7.483 & 2.679 & 12.375 & 7.441 & 2.715 & 9.317 & 0.042 & -0.037 & 0.018 \\
\hline 6 & 8.852 & 4.598 & 13.232 & 8.903 & 4.643 & 13.200 & -0.051 & -0.045 & 0.032 \\
\hline
\end{tabular}

TABLE 6: The difference between real coordinates and transformed coordinates using the inner parameter.

\begin{tabular}{|c|c|c|c|c|c|c|c|c|c|}
\hline \multirow[t]{2}{*}{ Target ball } & \multicolumn{3}{|c|}{$\begin{array}{l}\text { Measured value of the total } \\
\text { station }(\mathrm{m})\end{array}$} & \multicolumn{3}{|c|}{ Correction coordinate $(\mathrm{m})$} & \multicolumn{3}{|c|}{ Difference in coordinate $(\mathrm{m})$} \\
\hline & $X$ & $Y$ & $Z$ & $X$ & Y & $Z$ & $\delta X$ & $\delta Y$ & $\delta Z$ \\
\hline 5 & 7.483 & 2.679 & 12.375 & 7.491 & 2.669 & 12.358 & -0.008 & 0.009 & 0.016 \\
\hline 6 & 8.852 & 4.598 & 13.232 & 8.842 & 4.603 & 13.217 & 0.010 & -0.005 & 0.015 \\
\hline
\end{tabular}

TABLE 7: Comparison of position accuracy.

\begin{tabular}{lccc}
\hline Name & Error before correction $(\mathrm{mm})$ & Error after correction $(\mathrm{mm})$ & Improved accuracy $(\%)$ \\
\hline Error of $X$-direction & 47 & 9 & 80.9 \\
Error of $Y$-direction & 41 & 7 & 82.9 \\
Error of $Z$-direction & 25 & 16 & 36 \\
Accuracy & 67 & 20 & 70.1 \\
\hline
\end{tabular}

$$
R=\left[\begin{array}{cccc}
-0.045 & -0.252 & 0.967 & 0 \\
0.873 & -0.480 & -0.85 & 0 \\
0.485 & 0.849 & 0.242 & 0 \\
0 & 0 & 0 & 1
\end{array}\right] \cdot\left[\begin{array}{c}
\Delta X \\
\Delta Y \\
\Delta Z \\
1
\end{array}\right]=\left[\begin{array}{c}
5.984 \\
3.873 \\
6.098 \\
1
\end{array}\right]
$$

Using the calculated external orientation parameters, the scan coordinates of the other two target balls were converted to the coordinate system of the total station, and the difference between them is calculated (Table 5).

The error in each direction is as follows :

$$
\left.\begin{array}{l}
\sigma_{x}=0.047 \mathrm{~m} \\
\sigma_{y}=0.041 \mathrm{~m} \\
\sigma_{z}=0.025 \mathrm{~m}
\end{array}\right\} .
$$

The mean square error of measurement points is as follows:

$$
\sigma_{p}=\sqrt{\sigma_{x}^{2}+\sigma_{y}^{2}+\sigma_{z}^{2}}=0.067 \mathrm{~m}
$$

4.2. Correcting Error. By evaluating the external directional parameters and combining the internal parameters 
determined by the calibration platform of the $3 \mathrm{D}$ laser scanner, the coordinates of the standard test target ball center obtained by the 3D laser scanner can be converted into the coordinate system of the total station, and the difference between the measured coordinates and the corrected coordinates can be calculated (Table 6).

The error in each direction is as follows:

$$
\left.\begin{array}{rl}
\sigma_{x} & =0.009 \mathrm{~m} \\
\sigma_{y} & =0.007 \mathrm{~m} \\
\sigma_{z} & =0.016 \mathrm{~m}
\end{array}\right\} .
$$

The mean square error of measurement points is as follows :

$$
\sigma_{p}=\sqrt{\sigma_{x}^{2}+\sigma_{y}^{2}+\sigma_{z}^{2}}=0.020 \mathrm{~m} .
$$

Table 7 reflects the point position accuracy comparison before and after the error correction of the 3D laser scanner.

After the correction of the instrument system error, the errors in all directions of the test point were improved compared with those before the correction. Meanwhile, the error in the point position was increased from $67 \mathrm{~mm}$ before correction to $20 \mathrm{~mm}$, and the accuracy was increased by approximately $70.1 \%$.

\section{Conclusions}

To ensure that the accuracy of the 3D laser scanner fulfills the requirements, this study innovatively uses the $\mathrm{D}-\mathrm{H}$ modeling method in the robotics field to derive the error model of the 3D laser scanner, along with a detailed analysis of the six external orientation parameters and seven internal structure parameters. All these parameters are involved in the coordinate conversion error of the device, machining error, electrical installation error, and tooling error. In addition, this study integrally analyzes the error sources and whether the concrete measurement results are correct; experiments revealed that the D-H error model and calibration method proposed could enhance the instrument's accuracy significantly. Of note, the method proposed in this study analyzes most error sources, but does not consider the environmental temperature, air pressure, reflectivity, and other factors. How to integrate these factors to further enhance the accuracy of the system would be the next research direction.

\section{Data Availability}

The data that support the findings of this study are available from the corresponding author upon reasonable request.

\section{Conflicts of Interest}

The authors declare that they have no conflicts of interest.

\section{Acknowledgments}

This work was financially supported by the National Key Research and Development Program of China (grant nos. 2018YFE0121000 and 2020YFE0202800).

\section{References}

[1] Y. L. Guan, X. J. Cheng, X. W. Zhan, and T. D. Lu, "Research on systematic errors calibration of terrestrial laser scanner," Acta Geodaetica et Cartographica Sinica, vol. 43, no. 7, pp. 731-738, 2014.

[2] Z. L. Yu, Y. N. Yang, and L. Q. Zhu, "Study on calibration method for 3D laser scanning systems," Journal of Electronic Measurement and Instrument, vol. 21, no. 6, pp. 31-35, 2007.

[3] W. S. Jing and A. Y. Sun, "Research on accuracy evaluation method of ground 3D laser scanner," Geomatics \& Spatial Information Technology, vol. 39, no. 11, pp. 198-206, 2016.

[4] G. S. Cheok, S. D. Leigh, and A. R. Rukhin, "Calibration experiments of a laser scanner," 2002, http://www.nist.gov/ manuscript-publication-search.cfm?pub_id=860455.

[5] D. Barber, J. Mills, and S. Smith-Voysey, "Geometric validation of a ground-based mobile laser scanning system," ISPRS Journal of Photogrammetry and Remote Sensing, vol. 63, no. 1, pp. 128-141, 2008.

[6] D. H. Zheng, Y. Z. Sheng, and C. Liu, "3D laser scanner and its effect factor analysis of surveying error," Engineering of Surveying and Mapping, vol. 14, no. 2, pp. 32-34, 2005.

[7] D. Wang, X. S. Lu, and Z. J. Zhang, "Precision evaluation of RIEGL LMS-Q140i-80 laser scanner," Surveying and Mapping Science, vol. 31, no. 5, pp. 115-116, 2006.

[8] F. Gielsdorf, A. Rietdorf, and L. A. Gruendig, Concept for the Calibration of Terrestrial Laser Scanners: TS26 Positioning and Measurement Technologies and Practices II-Laser Scanning and Photogrammetry, FIG, Athens, Greece, 2004.

[9] D. D. Lichti and J. Franke, Self-Calibration of the iQsun 880 Laser Scanner, Optical 3D Measurement Techniques VII, Vienna, Austria, 2005.

[10] D. D. Lichti and M. G. Licht, "Experiences with terrestrial laser scanner modeling and accuracy assessment," in Proceedings of the ISPRS Commission V Symposium: Image Engineering and Vision Metrology: XXXVI, pp. 155-160, Dresden, Germany, September 2006.

[11] H. Q. Li, X. F. Yang, and Y. G. Zhao, "Precision factors and their control method for terrestrial 3D laser," Scanning Survey. Standardization of Surveying and Mapping, vol. 27, no. 1, pp. 29-31, 2006.

[12] W. X. Chen and Q. Yuan, "The research on self-calibration models of terrestrial laser scanner," Bulletin of Surveying and Mapping, vol. 59, no. 2, pp. 25-27, 2013.

[13] Y. Reshetyuk, "Calibration of terrestrial laser scanners for the purpose of geodetic engineering," in Proceedings of the $3 \mathrm{rd}$ IAG/12th FIG Symposium, Baden Germany, May 2006.

[14] J. Zhang and W. S. Jiang, "Self-calibration of LiDAR system mounting biases using virtual tie point model," Acta Geodaetica et Cartographical Sinica, vol. 40, no. 6, pp. 762-769, 2011.

[15] W. X. Xu, Z. W. Tian, Z. Zhou et al., "Calibration of mounted parameter for ship-borne 3D laser scanning system," Acta Geodaetica et Cartographical Sinica, vol. 47, no. 2, pp. 208214, 2018.

[16] Y. M. Han, L. Z. Wang, and X. S. Lu, "Angle error calibration of $360^{\circ}$ laser scanner," Geotechnical Investigation \& Surveying, vol. 2, pp. 59-63, 2011.

[17] S. Z. Xu, Research on Calibration of Mobile Mapping System for Land Vehicle and its Accuracy Assessment, Wuhan University, Wuhan, China, 2016.

[18] Y. Sun, G. Li, N. Zhang, Q. Chang, J. Xu, and J. Zhang, "Development of ensemble learning models to evaluate the 
strength of coal-grout materials," International Journal of Mining Science and Technology, vol. 31, 2020.

[19] Y. Sun, G. Li, H. Basarir, A. Karrech, and M. R. Azadi, "Laboratory evaluation of shear strength properties for cement-based grouted coal mass," Arabian Journal of Geosciences, vol. 12, no. 22, p. 690, 2019.

[20] X. Song, J. Chen, and J. Yu, "Polishing robot kinematics model based on the D-H method," Electronic Science and Technology, vol. 30, no. 6, pp. 50-53, 2017.

[21] X. Wu and D. Zheng, "Research on multiple measurement models based on D-H matrix in flexible coordinate measuring machine," Journal of Electronic Measurement and Instrumentation, vol. 29, no. 5, pp. 760-764, 2015. 